\title{
CONVERGENCE RATE IN THE WEIGHTED NORM FOR A SEMILINEAR HEAT EQUATION WITH SUPERCRITICAL NONLINEARITY
}

\author{
YŪKI NAITO
}

\begin{abstract}
We study the behavior of solutions to the Cauchy problem for a semilinear heat equation with supercritical nonlinearity. It is known that two solutions approach each other if these initial data are close enough near the spatial infinity. In this paper, we give its sharp convergence rate in the weighted norms for a class of initial data. Proofs are given by a comparison method based on matched asymptotics expansion.
\end{abstract}

\section{Introduction}

We consider the Cauchy problem

$$
\begin{cases}u_{t}=\Delta u+|u|^{p-1} u, & x \in \mathbf{R}^{N}, t>0, \\ u(x, 0)=u_{0}(x), & x \in \mathbf{R}^{N},\end{cases}
$$

where $u=u(x, t), \Delta$ is the Laplacian, $p>1$ and $u_{0}$ is a given continuous function on $\mathbf{R}^{N}$ that decays to zero as $|x| \rightarrow \infty$. equation

We first recall some known facts concerning positive solutions of the elliptic

$$
\Delta \phi+\phi^{p}=0 \text { in } \mathbf{R}^{N}
$$

with $N \geq 3$. It is well known that there exists a classical positive radial solution of (1.2) if and only if $p \geq(N+2) /(N-2)$. (See, e.g., [1,4].) We denote by $\phi_{\alpha}$ a solution of the problem

$$
\left\{\begin{array}{l}
\phi_{r r}+\frac{N-1}{r} \phi_{r}+\phi^{p}=0 \text { for } r>0, \\
\phi(0)=\alpha \text { and } \phi_{r}(0)=0 .
\end{array}\right.
$$

2010 Mathematics Subject Classification. 35K15; 35B35; 35B40.

Key words and phrases. Semilinear heat equation, Convergence rate, Supercirtical exponent.

This research was supported by the Grant-in-Aid for Scientific Research (C) (No. 23540244) Japan Society for the Promotion of Science.

Received March 12, 2014; revised May 14, 2014. 
If $p \geq(N+2) /(N-2)$ then, for each $\alpha>0, \phi_{\alpha}(r)$ is positive and strictly decreasing for $r \geq 0$, and satisfies $\phi_{\alpha}(r) \rightarrow 0$ as $r \rightarrow \infty$.

Define the exponent $p_{c}$ by

$$
p_{c}= \begin{cases}\frac{(N-2)^{2}-4 N+8 \sqrt{N-1}}{(N-2)(N-10)}, & N \geq 11, \\ \infty, & 3 \leq N \leq 10 .\end{cases}
$$

The exponent $p_{c}$ has appeared in several different studies of (1.1) and related problems, see, e.g., $[8,11,5,6]$. It was shown by Wang [11] that, for $(N+2) /$ $(N-2) \leq p<p_{c}$, each pair of positive radial solutions of (1.2) intersect each other, and that, for $p \geq p_{c}$, these solutions are strictly ordered such that $\phi_{\alpha}(r)$ is strictly increasing in $\alpha$ for each $r$ and satisfies

$$
\lim _{\alpha \rightarrow \infty} \phi_{\alpha}(r)=\phi_{\infty}(r) \text { for } r>0
$$

where $\phi_{\infty}$ a singular solution given by

$$
\phi_{\infty}(r)=L r^{-m} \text { for } r>0
$$

with

$$
m=\frac{2}{p-1} \quad \text { and } \quad L=(m(N-2-m))^{1 /(p-1)} .
$$

It was also shown in [5] that, for $p>p_{c}$, the solution $\phi_{\alpha}$ has the expansion

$$
\phi_{\alpha}(|x|)=L|x|^{-m}+a(\alpha)|x|^{-m-\lambda_{1}}+o\left(|x|^{-m-\lambda_{1}}\right) \quad \text { as }|x| \rightarrow \infty,
$$

where $\lambda_{1}$ is a positive constant given by

$$
\lambda_{1}=\lambda_{1}(N, p)=\frac{N-2-2 m-\sqrt{(N-2-m)^{2}-8(N-2-m)}}{2},
$$

and $a(\alpha)$ is a positive number. Note that $\lambda_{1}$ is a smaller root of the quadratic polynomial

$$
\lambda^{2}-(N-2-2 m) \lambda+2(N-2-m)=0 .
$$

We denote by

$$
\lambda_{2}=\lambda_{2}(N, p)=\frac{N-2-2 m+\sqrt{(N-2-m)^{2}-8(N-2-m)}}{2}
$$

a larger root of the quadratic polynomial.

For $\ell \geq 0$, we define the weighted norm

$$
\|\psi\|_{\ell}=\sup _{x \in \mathbf{R}^{N}}(1+|x|)^{\ell}|\psi(x)|,
$$

where $\psi$ be a continuous function on $\mathbf{R}^{N}$. It is clear that $\|\cdot\|_{\ell}=\|\cdot\|_{L^{\infty}\left(\mathbf{R}^{N}\right)}$ if $\ell=0$. 
The following results have been proved by $[5$, Theorem 1.15] and $[6$, Theorem 2].

THEOREM A. Let $p>p_{c}$ and $\alpha>0$.

(i) The stationary solution $\phi_{\alpha}$ is stable with respect to $\|\cdot\|_{m+\lambda_{1}}$, that is, for any $\varepsilon>0$ there is $\delta>0$ such that, if $\left\|u_{0}-\phi_{\alpha}\right\|_{m+\lambda_{1}}<\delta$, then the solution $u$ of (1.1) satisfies $\left\|u(\cdot, t)-\phi_{\alpha}\right\|_{m+\lambda_{1}}<\varepsilon$ for all $t>0$.

(ii) Let $\ell \in\left(m+\lambda_{1}, m+\lambda_{2}\right]$. Then $\phi_{\alpha}$ is stable with respect to $\|\cdot\|_{\ell}$ and there exists $\delta>0$ such that, if $\left\|u_{0}-\phi_{\alpha}\right\|_{\ell}<\delta$, then the solution $u$ of (1.1) satisfies $\left\|u(\cdot, t)-\phi_{\alpha}\right\|_{\ell^{\prime}} \rightarrow 0$ as $t \rightarrow \infty$ for any $\ell^{\prime} \in(0, \ell)$.

Polácik and Yanagida [10] improved the above results and proved that the solutions approach a set of stationary solutions for a wide class of initial data. Later, Fila, Winkler and Yanagida [3] and Hoshino and Yanagida [7] studied more general problem. Denote by $u$ and $\tilde{u}$ solutions of (1.1) with initial data $u_{0}$ and $\tilde{u}_{0}$, respectively. In $[3,7]$ they showed that how fast two solutions $u$ and $\tilde{u}$ approach each other as $t \rightarrow \infty$ if $u_{0}$ and $\tilde{u}_{0}$ are close enough near the spatial infinity. Clearly, in the case $\tilde{u}_{0}(x)=\phi_{\alpha}(|x|)$, the rate of approach of these solutions corresponds to the convergence rate to the steady state. Precisely, the following results were shown by $[3,7]$.

Theorem B. Let $p>p_{c}$ and $m+\lambda_{1}<\ell<m+\lambda_{2}+2$. Assume that $u_{0}$ and $\tilde{u}_{0}$ satisfy

$$
-\phi_{\alpha}(|x|) \leq u_{0}(x), \quad \tilde{u}_{0}(x) \leq \phi_{\alpha}(|x|) \quad \text { for } x \in \mathbf{R}^{N}
$$

with some $\alpha>0$. If

$$
\limsup _{|x| \rightarrow \infty}|x|^{\ell}\left|u_{0}(x)-\tilde{u}_{0}(x)\right|<\infty,
$$

then the solutions $u$ and $\tilde{u}$ of (1.1) satisfy

$$
\limsup _{t \rightarrow \infty} t^{\left(\ell-m-\lambda_{1}\right) / 2}\|u(\cdot, t)-\tilde{u}(\cdot, t)\|_{L^{\infty}\left(\mathbf{R}^{N}\right)}<\infty .
$$

In this paper, we consider the optimal rate of approach of these solutions in the weighted norm $\|\cdot\|_{\ell}$, and verify that the rate depends on the order $\ell$. We also show the stability of solutions with respect to the norm $\|\cdot\|_{\ell}$ for $m+\lambda_{1}<$ $\ell<m+\lambda_{2}+2$.

Our first result is the following.

THEOREM 1.1. Let $p>p_{c}$ and $\ell \in\left(m+\lambda_{1}, m+\lambda_{2}+2\right)$. Assume that $u_{0}, \tilde{u}_{0} \in C\left(\mathbf{R}^{N}\right)$ satisfy (1.4) with some $\alpha>0$. If (1.5) holds, then, for $\ell^{\prime} \in[0, \ell)$, the solutions $u$ and $\tilde{u}$ of (1.1) satisfy

$$
\limsup _{t \rightarrow \infty} t^{v}\|u(\cdot, t)-\tilde{u}(\cdot, t)\|_{\ell^{\prime}}<\infty,
$$


where

$$
v= \begin{cases}\left(\ell-m-\lambda_{1}\right) / 2 & \text { if } 0 \leq \ell^{\prime} \leq m+\lambda_{1}, \\ \left(\ell-\ell^{\prime}\right) / 2 & \text { if } m+\lambda_{1}<\ell^{\prime}<\ell .\end{cases}
$$

Furthermore, for any constants $c>0$ and $\mu \geq 1 / 2$, the solutions $u$ and $\tilde{u}$ satisfy

$$
\limsup _{t \rightarrow \infty} t^{\left(\ell-\ell^{\prime}\right) \mu}\left(\sup _{|x| \geq c t^{\mu}}(1+|x|)^{\ell^{\prime}}|u(x, t)-\tilde{u}(x, t)|\right)<\infty .
$$

The next result shows that the upper estimates given in Theorem 1.1 are optimal.

THeOREM 1.2. Let $p>p_{c}$ and $\ell \in\left(m+\lambda_{1}, m+\lambda_{2}+2\right)$. Assume that $u_{0}, \tilde{u}_{0} \in C\left(\mathbf{R}^{N}\right)$ satisfy

$$
\phi_{\alpha}(|x|) \leq \tilde{u}_{0}(x)<u_{0}(x) \leq \phi_{\infty}(|x|) \quad \text { for } x \in \mathbf{R}^{N} \backslash\{0\}
$$

with some $\alpha>0$. If

$$
\liminf _{|x| \rightarrow \infty}|x|^{\ell}\left(u_{0}(x)-\tilde{u}_{0}(x)\right)>0,
$$

then, for $\ell^{\prime} \in[0, \ell)$, the solutions $u$ and $\tilde{u}$ of (1.1) satisfy

$$
\liminf _{t \rightarrow \infty} t^{v}\|u(\cdot, t)-\tilde{u}(\cdot, t)\|_{\ell^{\prime}}>0,
$$

where $v$ is the constant defined by (1.7). Furthermore, the following (i) and (ii) hold.

(i) For any constant $c>0$, the solutions $u$ and $\tilde{u}$ satisfy

$$
\liminf _{t \rightarrow \infty} t^{\left(\ell-m-\lambda_{1}\right) / 2}\left(\inf _{|x| \leq c t^{1 / 2}}(1+|x|)^{m+\lambda_{1}}|u(x, t)-\tilde{u}(x, t)|\right)>0 .
$$

(ii) Let $\ell^{\prime} \in[0, \ell)$. For any constants $c>0$ and $\mu \geq 1 / 2$, the solutions $u$ and $\tilde{u}$ satisfy

$$
\liminf _{t \rightarrow \infty} t^{\left(\ell-\ell^{\prime}\right) \mu}\left(\sup _{|x| \geq c t^{\mu}}(1+|x|)^{\ell^{\prime}}|u(x, t)-\tilde{u}(x, t)|\right)>0 .
$$

Remark 1.1. In Theorem 1.2, we also assume that $u_{0}(x), \tilde{u}_{0}(x) \leq \phi_{\tilde{\alpha}}(|x|)$ for $x \in \mathbf{R}^{N}$ with some $\tilde{\alpha}>\alpha$, and that (1.5) holds. Then, by applying Theorem 1.1 with $\alpha=\tilde{\alpha}$, we obtain (1.6) with (1.7) and (1.8). Thus, from (1.6) with $\ell^{\prime}=$ $m+\lambda_{1}$, we obtain, for any constant $c>0$,

$$
\limsup _{t \rightarrow \infty} t^{\left(\ell-m-\lambda_{1}\right) / 2}\left(\sup _{|x| \leq c t^{1 / 2}}(1+|x|)^{m+\lambda_{1}}(u(x, t)-\tilde{u}(x, t))\right)<\infty .
$$


Thus, in the range $|x| \leq c t^{1 / 2}$, the solutions $u$ and $\tilde{u}$ approach uniformly in the senses (1.12) and (1.14). In particular, for any constant $c>0$, we have

$$
\begin{aligned}
0 & <\liminf _{t \rightarrow \infty} t^{\left(\ell-m-\lambda_{1}\right) / 2}\left(\inf _{|x| \leq c}(u(x, t)-\tilde{u}(x, t))\right) \\
& \leq \limsup _{t \rightarrow \infty} t^{\left(\ell-m-\lambda_{1}\right) / 2}\left(\sup _{|x| \leq c}(u(x, t)-\tilde{u}(x, t))\right)<\infty .
\end{aligned}
$$

On the other hand, in the range $|x| \geq c t^{\mu}$ with $\mu>1 / 2$, the solutions approaches each other in the different rate by (1.8) and (1.13). In particular, for any constant $c>0$, we obtain

$$
\begin{aligned}
0 & <\liminf _{t \rightarrow \infty} t^{\left(\ell-\ell^{\prime}\right) / 2}\left(\sup _{|x| \geq c t^{1 / 2}}(1+|x|)^{\ell^{\prime}}(u(x, t)-\tilde{u}(x, t))\right) \\
& \leq \limsup _{t \rightarrow \infty} t^{\left(\ell-\ell^{\prime}\right) / 2}\left(\sup _{|x| \geq c t^{1 / 2}}(1+|x|)^{\ell^{\prime}}(u(x, t)-\tilde{u}(x, t))\right)<\infty .
\end{aligned}
$$

Note here that the constant $v$ defined by (1.7) fulfills the property that $v=$ $\min \left\{\left(\ell-m-\lambda_{1}\right) / 2,\left(\ell-\ell^{\prime}\right) / 2\right\}$.

We consider the stability of solutions with respect to the norm $\|\cdot\|_{\ell}$.

THEOREM 1.3. Let $p>p_{c}$ and $\ell \in\left(m+\lambda_{1}, m+\lambda_{2}+2\right)$.

(i) Assume that $u_{0}, \tilde{u}_{0} \in C\left(\mathbf{R}^{N}\right)$ satisfy (1.4) with some $\alpha>0$. For any $\varepsilon>0$ there exists $\delta>0$ such that, if $\left\|u_{0}(\cdot)-\tilde{u}_{0}(\cdot)\right\|_{\ell}<\delta$, then the solutions $u$ and $\tilde{u}$ of (1.1) satisfy $\|u(\cdot, t)-\tilde{u}(\cdot, t)\|_{\ell}<\varepsilon$ for all $t \geq 0$.

(ii) Assume that $u_{0}$ and $\tilde{u}_{0}$ satisfy (1.9) with some $\alpha>0$. If (1.10) holds, then the solutions $u$ and $\tilde{u}$ satisfy

$$
\liminf _{t \rightarrow \infty}\|u(\cdot, t)-\tilde{u}(\cdot, t)\|_{\ell}>0 .
$$

Let us consider the convergence rate of solutions to the steady state. Assume that $u_{0} \in C\left(\mathbf{R}^{N}\right)$ satisfies

$$
-\phi_{\infty}(x)<u_{0}(x)<\phi_{\infty}(x) \text { for } x \in \mathbf{R}^{N} \backslash\{0\} .
$$

and

$$
\limsup _{|x| \rightarrow \infty}|x|^{\ell}\left|u_{0}(x)-\phi_{\alpha}(|x|)\right|<\infty
$$

with some $\alpha>0$. Then there exists $\beta>\alpha$ such that $-\phi_{\beta}(x) \leq u_{0}(x) \leq \phi_{\beta}(x)$ for $x \in \mathbf{R}^{N}$. Applying Theorem 1.1 with $\alpha=\beta$ and $\tilde{u}_{0}=\phi_{\alpha}$, and Theorem 1.2 with $\tilde{u}_{0}=\phi_{\alpha}$, we obtain the following 
Corollary 1.1. Let $p>p_{c}$ and $\ell \in\left(m+\lambda_{1}, m+\lambda_{2}+2\right)$. Assume that $u_{0} \in C\left(\mathbf{R}^{N}\right)$ satisfies (1.16) and (1.17) with some $\alpha>0$. Then the solution $u$ of (1.1) satisfies

$$
\left\|u(\cdot, t)-\phi_{\alpha}(|\cdot|)\right\|_{\ell^{\prime}} \rightarrow 0 \quad \text { as } t \rightarrow \infty
$$

for any $\ell^{\prime} \in[0, \ell)$. Precisely, the solution $u$ satisfies

$$
\limsup _{t \rightarrow \infty} t^{v}\left\|u(\cdot, t)-\phi_{\alpha}(|\cdot|)\right\|_{\ell^{\prime}}<\infty,
$$

where $v$ is the constant defined by (1.7). Furthermore, if $u_{0}$ satisfies $u_{0}(x)>$ $\phi_{\alpha}(|x|)$ for $x \in \mathbf{R}^{N}$ and

$$
\liminf _{|x| \rightarrow \infty}|x|^{\ell}\left(u_{0}(x)-\phi_{\alpha}(|x|)\right)>0,
$$

then the solution $u$ satisfies

$$
\liminf _{t \rightarrow \infty} t^{v}\left\|u(\cdot, t)-\phi_{\alpha}(|\cdot|)\right\|_{\ell^{\prime}}>0
$$

for $\ell^{\prime} \in[0, \ell)$ with the constant $v$ defined by (1.7).

We consider the stability of the steady state with respect to the norm $\|\cdot\|_{\ell}$ by applying Theorem 1.3. Let $p>p_{c}$ and $\alpha>0$. Put $\beta>\alpha$. Then there exists $\tilde{\delta}=\tilde{\delta}(\beta)>0$ such that, if $\left\|u_{0}(\cdot)-\phi_{\alpha}(|\cdot|)\right\|_{\ell}<\tilde{\delta}$, then $-\phi_{\beta}(|x|) \leq u_{0}(x) \leq \phi_{\beta}(|x|)$ for $x \in \mathbf{R}^{N}$. Applying Theorem 1.3 (i) with $\alpha=\beta, \tilde{u}_{0}=\phi_{\alpha}$, and $\min \{\delta, \tilde{\delta}\}$ instead of $\delta$, and Theorem 1.3 (ii) with $\tilde{u}_{0}=\phi_{\alpha}$, we obtain the following

Corollary 1.2. Let $p>p_{c}, \alpha>0$, and let $\ell \in\left(m+\lambda_{1}, m+\lambda_{2}+2\right)$. For any $\varepsilon>0$ there exists $\delta>0$ such that, if $\left\|u_{0}(\cdot)-\phi_{\alpha}(|\cdot|)\right\|_{\ell}<\delta$, then the solution $u$ of $(1.1)$ satisfy $\left\|u(\cdot, t)-\phi_{\alpha}(|\cdot|)\right\|_{\ell}<\varepsilon$ for all $t \geq 0$. Furthermore, if $u_{0}$ satisfies $u_{0}(x)>\phi_{\alpha}(|x|)$ for $x \in \mathbf{R}^{N}$ and (1.19), then the solution $u$ satisfy

$$
\liminf _{t \rightarrow \infty}\left\|u(\cdot, t)-\phi_{\alpha}(|\cdot|)\right\|_{\ell}>0 \text {. }
$$

Remark 1.2. (i) By (1.20) we can not expect that (1.18) holds with $\ell^{\prime}=\ell$. (ii) It was shown in [9] that, for any $\varepsilon>0$, there exists $\delta>0$ such that, if

$$
\limsup _{|x| \rightarrow \infty}|x|^{m+\lambda_{1}}\left|u_{0}(x)-\phi_{\alpha}(|x|)\right|<\delta
$$

then the solution $u$ of $(1.1)$ satisfies $\lim \sup _{t \rightarrow \infty}\left\|u(\cdot, t)-\phi_{\alpha}(|\cdot|)\right\|_{m+\lambda_{1}} \leq \varepsilon$. In the case $\ell>m+\lambda_{1}$, it is an open and interesting question, for any $\varepsilon>0$, whether there exists $\delta>0$ such that, if

$$
\limsup _{|x| \rightarrow \infty}|x|^{\ell}\left|u_{0}(x)-\phi_{\alpha}(|x|)\right|<\delta,
$$

then $\lim \sup _{t \rightarrow \infty}\left\|u(\cdot, t)-\phi_{\alpha}(|\cdot|)\right\|_{\ell}<\varepsilon$ holds. 
For the attractivity property of steady states and its convergence rate in the norm $\|\cdot\|_{\ell}$ with $\ell=m+\lambda_{1}$, we refer to [9].

Proofs of the above theorems are obtained by a comparison technique for the linearized equation. Our approach is mainly based on the ideas of $[3,7]$, we however need some additional ingredients to obtain the convergence properties in the weighted norms.

This paper is organized as follows. In Section 2 we recall some results of $[11,3]$ concerning super and sub-solution methods and certain linearized problems. In Sections 3 and 4, we give the proof of Theorems 1.1 and 1.2, respectively, by deriving suitable upper and lower bound for solutions of these linearized problems. Finally, in Section 5, we prove Theorem 1.3.

\section{Preliminary results}

We first recall the definition of continuous weak super and sub-solutions to the following general problem

$$
\begin{cases}u_{t}=\Delta u+f(|x|, u), & x \in \mathbf{R}^{N}, t>0 \\ u(x, 0)=u_{0}(x), & x \in \mathbf{R}^{N}\end{cases}
$$

where $f(r, u)$ is continuous on $([0, \infty) \times \mathbf{R})$, locally Hölder continuous in $r \in[0, \infty)$ locally uniformly with respect to $u \in \mathbf{R}$, and locally Lipschitz continuous in $u$ locally uniformly with respect to $r$. We say that $u$ is a continuous weak supersolution of (2.1) for $0 \leq t \leq T$ if $u$ is continuous on $\mathbf{R}^{N} \times[0, T], u(x, 0) \geq u_{0}(x)$ and satisfies, for any $\xi \in C^{2,1}\left(\mathbf{R}^{N} \times[0, T]\right)$ with $\xi \geq 0$ and $\operatorname{supp} \xi(\cdot, t)$ being compact in $\mathbf{R}^{N}$ for all $t \in[0, T]$,

$$
\left.\int_{\mathbf{R}^{N}} u(x, t) \xi(x, t) d x\right|_{t=0} ^{t=T^{\prime}} \geq \int_{0}^{T^{\prime}} \int_{\mathbf{R}^{N}} u(x, t)\left(\xi_{t}+\Delta \xi\right)(x, t)+f(|x|, u) \xi(x, t) d x d t
$$

for all $T^{\prime} \in[0, T]$. Continuous weak subsolutions are defined in a similar way by reversing the inequalities. Consider the corresponding elliptic equation

$$
\Delta u+f(|x|, u)=0 \quad \text { in } \mathbf{R}^{N} .
$$

We call a function $u$ a continuous weak supersolution of (2.2) in $\mathbf{R}^{N}$ if $u$ is continuous in $\mathbf{R}^{N}$ and satisfies, for any nonnegative function $\eta \in C_{0}^{\infty}\left(\mathbf{R}^{N}\right)$,

$$
\int_{\mathbf{R}^{N}} u(x) \Delta \eta(x)+f(|x|, u) \eta(x) d x \leq 0 .
$$

Continuous weak sub-solutions are defined in a similar way by reversing the inequality in (2.3).

The following results are shown by Wang [11].

Lemma 2.1. Let $\bar{u}$ and $\underline{u}$ be bounded continuous weak super and sub-solutions of (2.1), respectively. Then (2.1) has a unique classical solution $u$ with $\underline{u} \leq u \leq \bar{u}$ in $\mathbf{R}^{N} \times(0, \infty)$. 
We say that $\bar{u}$ is a classical supersolution of (2.1) if $\bar{u}$ satisfies

$$
\bar{u}_{t} \geq \Delta \bar{u}+f(|x|, \bar{u}) \text { for } x \in \mathbf{R}^{N}, t>0
$$

and $\bar{u}(x, 0) \geq u_{0}(x)$ for $x \in \mathbf{R}^{N}$. Classical subsolutions are defined in a similar way by reversing the inequalities. result.

By the similar argument as in [11, Proposition 3.8], we obtain the following

LEMmA 2.2. Let $r(t) \in(0, \infty)$ be a continuous function for $t \geq 0$, and define $D_{1}=\left\{(x, t) \in \mathbf{R}^{N} \times[0, \infty):|x|<r(t), t \geq 0\right\}$ and $D_{2}=\left\{(x, t) \in \mathbf{R}^{N} \times[0, \infty):|x|\right.$ $>r(t), t \geq 0\}$.

(i) Suppose that $u_{1}(r, t)$ and $u_{2}(r, t)$, with $r=|x|$, are classical supersolutions of (2.1) in $D_{1}$ and $D_{2}$, respectively. Assume that $u_{1}=u_{2}$ and $\partial u_{1} / \partial r \geq$ $\partial u_{2} / \partial r$ at $(r, t)=(r(t), t)$ for all $t \geq 0$. For each $t \geq 0$, put

$$
\bar{u}(r, t)= \begin{cases}u_{1}(r, t), & \text { for } 0 \leq r \leq r(t), \\ u_{2}(r, t), & \text { for } r>r(t) .\end{cases}
$$

Then $\bar{u}(|x|, t)$ is a continuous weak supersolution to $(2.1)$ in $\mathbf{R}^{N} \times[0, \infty)$.

(ii) Suppose that $v_{1}(r, t)$ and $v_{2}(r, t)$, with $r=|x|$, are classical sub-solutions of (2.1) in $D_{1}$ and $D_{2}$, respectively. Assume that $v_{1}=v_{2}$ and $\partial v_{1} / \partial r \leq$ $\partial v_{2} / \partial r$ at $(r, t)=(r(t), t)$ for all $t \geq 0$. For each $t \geq 0$, put

$$
\underline{u}(r, t)= \begin{cases}v_{1}(r, t), & \text { for } 0 \leq r \leq r(t), \\ v_{2}(r, t), & \text { for } r>r(t) .\end{cases}
$$

Then $\underline{u}(|x|, t)$ is a continuous weak subsolution to $(2.1)$ in $\mathbf{R}^{N} \times(0, \infty)$.

Proof. Since the proof of (ii) is similar to (i), we will show (i) only. Take any $\xi \in C^{2,1}\left(\mathbf{R}^{N} \times[0, T]\right)$ with $\xi \geq 0$ and $\operatorname{supp} \xi(\cdot, t)$ being compact in $\mathbf{R}^{N}$ for all $t \in[0, T]$. For each $i=1,2, u_{i}=u_{i}(x, t)$ satisfies

$$
\left(u_{i}\right)_{t} \geq \Delta u_{i}+f\left(|x|, u_{i}\right), \quad(x, t) \in D_{i} .
$$

Fix $t \in(0, T)$ arbitrarily. Multiplying $(2.4)_{1}$ by $\xi(x, t)$, and integrating it on $\{x:|x|<r(r)\}$, we obtain

$$
\int_{|x|<r(t)}\left(u_{1}\right)_{t} \xi d x \geq \int_{|x|<r(t)}\left(\Delta u_{1}\right) \xi d x+\int_{|x|<r(t)} f\left(|x|, u_{1}\right) \xi d x .
$$

By using of the integration by parts, we have

$$
\int_{|x|<r(t)}\left(\Delta u_{1}\right) \xi d x=\int_{|x|=r(t)}\left(\xi \frac{\partial u_{1}}{\partial n}-u_{1} \frac{\partial \xi}{\partial n}\right) d S+\int_{|x|<r(t)} u_{1}(\Delta \xi) d x
$$

where $n$ is the outward unit normal vector to $|x|=r(t)$ and $d S$ denotes the surface measure on $|x|=r(t)$. Thus we obtain 


$$
\begin{aligned}
\int_{|x|<r(t)}\left(u_{1}\right)_{t} \xi d x \geq & \int_{|x|=r(t)}\left(\xi \frac{\partial u_{1}}{\partial n}-u_{1} \frac{\partial \xi}{\partial n}\right) d S \\
& +\int_{|x|<r(t)}\left(u_{1}(\Delta \xi)+f\left(|x|, u_{1}\right) \xi\right) d x
\end{aligned}
$$

Multiplying $(2.4)_{2}$ by $\xi$, and integrating by parts on $\{x:|x|>r(t)\}$, we obtain

$$
\begin{aligned}
\int_{|x|>r(t)}\left(u_{2}\right)_{t} \xi d x \geq & \int_{|x|=r(t)}\left(-\xi \frac{\partial u_{2}}{\partial n}+u_{2} \frac{\partial \xi}{\partial n}\right) d S \\
& +\int_{|x|>r(t)}\left(u_{2}(\Delta \xi)+f\left(|x|, u_{2}\right) \xi\right) d x
\end{aligned}
$$

Adding (2.5) and (2.6), we obtain

$$
\int_{\mathbf{R}^{N}}(\bar{u})_{t} \xi d x \geq \int_{|x|=r(t)} \xi\left(\frac{\partial u_{1}}{\partial n}-\frac{\partial u_{2}}{\partial n}\right) d S+\int_{\mathbf{R}^{N}}(\bar{u}(\Delta \xi)+f(|x|, \bar{u}) \xi) d x .
$$

Since $\partial u_{1} / \partial r \geq \partial u_{2} / \partial r$ on $r=r(t)$, we have $\partial u_{1} / \partial n-\partial u_{2} / \partial n \geq 0$ on $|x|=r(t)$. Thus we obtain

$$
\int_{\mathbf{R}^{N}}(\bar{u})_{t} \xi d x \geq \int_{\mathbf{R}^{N}}(\bar{u}(\Delta \xi)+f(|x|, \bar{u}) \xi) d x
$$

Integrating by parts on $t \in\left[0, T^{\prime}\right]$, we obtain

$$
\left.\int_{\mathbf{R}^{N}} \bar{u}(|x|, t) \xi(x, t) d x\right|_{t=0} ^{t=T^{\prime}} \geq \int_{0}^{T^{\prime}} \int_{\mathbf{R}^{N}}\left(\bar{u}(|x|, t)\left(\xi_{t}+\Delta \xi\right)(x, t)+f(|x|, \bar{u}) \xi(x, t)\right) d x d t .
$$

Since $u_{1}$ and $u_{2}$ are classical supersolution of (2.1), we have $\bar{u}(|x|, t) \geq u_{0}(x)$ for $x \in \mathbf{R}^{N}$. Thus $\bar{u}$ is a continuous weak supersolution of $(2.1)$.

We next summarize previous results of [3] on the problem for a linearized equation of (1.1) at a steady state $\phi_{\alpha}$. For $\alpha>0$ we define the linear operator $P_{\alpha}$ by

$$
P_{\alpha} U=U_{r r}+\frac{N-1}{r} U_{r}+p \phi_{\alpha}(r)^{p-1} U
$$

and consider the solutions $U=U(r, t)$ of the problem

$$
\begin{cases}U_{t}=P_{\alpha} U & \text { for } r>0, t>0 \\ U_{r}(0, t)=0 & \text { for } t>0 \\ U(r, 0)=U_{0}(r) & \text { for } r \geq 0\end{cases}
$$

where $U_{0}(r)$ is a continuous function decaying to zero as $r \rightarrow \infty$. By the maximum principle, we see that $U(\cdot, t)>0$ for all $t>0$ if $U_{0} \geq 0$ and $U_{0} \not \equiv 0$. 
Let $\psi(r)$ satisfy

$$
\begin{cases}P_{\alpha} \psi=0 & \text { for } r>0, \\ \psi(0)=1 & \text { and } \psi_{r}(0)=0,\end{cases}
$$

and let $\Psi$ denote the solution to

$$
\begin{cases}P_{\alpha} \Psi=\psi & \text { for } r>0, \\ \Psi(0)=0 & \text { and } \Psi_{r}(0)=0 .\end{cases}
$$

We state some useful properties of $\psi$, which were proved in [3, Lemma 2.3].

Lemma 2.3. Let $p>p_{c}$ and $\alpha>0$. Then the solution $\psi$ of (2.8) is given by $\psi(r)=\partial \phi_{\alpha}(r) / \partial \alpha$ for $r \geq 0, \psi(r)$ is positive for all $r \geq 0$, and the solution $\Psi(r)$ of (2.9) is positive for all $r>0$. Moreover, $\psi(r)$ is decreasing for $r>0$ and satisfies

$$
\lim _{r \rightarrow \infty} r^{m+\lambda_{1}} \psi(r)=c_{\alpha}
$$

for some positive constant $c_{\alpha}$.

We recall comparison results in [3, Lemmas 2.1 and 2.2]. Let $u$ and $\tilde{u}$ denote solutions of (1.1) with initial data $u_{0}, \tilde{u}_{0}$, respectively.

Lemma 2.4. Let $p \geq p_{c}$ and $\alpha>0$.

(i) Assume that $u_{0}$ and $\tilde{u}_{0}$ satisfy

$$
-\phi_{\alpha}(x) \leq u_{0}(x), \tilde{u}_{0}(x) \leq \phi_{\alpha}(x) \text { for } x \in \mathbf{R}^{N} .
$$

If $\left|u_{0}(x)-\tilde{u}_{0}(x)\right| \leq U_{0}(|x|)$ for $x \in \mathbf{R}^{N}$, then the solutions $U$ of (2.7) and $u, \tilde{u}$ of (1.1) satisfy

$$
|u(x, t)-\tilde{u}(x, t)| \leq U(|x|, t) \text { for all } x \in \mathbf{R}^{N}, t>0 .
$$

(ii) Assume that $u_{0}$ and $\tilde{u}_{0}$ satisfy

$$
\phi_{\alpha}(|x|) \leq \tilde{u}_{0}(x) \leq u_{0}(x) \leq \phi_{\infty}(|x|) \quad \text { for } x \in \mathbf{R}^{N} \backslash\{0\} .
$$

If $u_{0}(x)-\tilde{u}_{0}(x) \geq U_{0}(|x|) \geq 0$ for $x \in \mathbf{R}^{N}$, then the solutions $U$ of (2.7) and $u, \tilde{u}$ of (1.1) satisfy

$$
u(x, t)-\tilde{u}(x, t) \geq U(|x|, t) \geq 0 \text { for all } x \in \mathbf{R}^{N}, t>0 .
$$

\section{Proof of Theorem 1.1}

In the proof of Theorem 1.1, we construct suitable super- and subsolutions of (2.7). We use the ideas presented in [3,7], and this section is similar to Section 3 in [7]. But in order to obtain the convergence rate in the weighted norms, we need some additional ideas.

We first construct a supersolution of (2.7). We recall the result by [2, Lemma 3.1]. 
LEMMA 3.1. If $m+\lambda_{1}<\ell<m+\lambda_{2}+2$, then there exists a positive solution $F$ of

$$
F_{\eta \eta}+\frac{N-1}{\eta} F_{\eta}+\frac{\eta}{2} F_{\eta}+\frac{\ell}{2} F+\frac{p L^{p-1}}{\eta^{2}} F=0 \text { for } \eta>0
$$

satisfying

$$
\lim _{\eta \rightarrow 0} \eta^{m+\lambda_{1}} F(\eta)=a_{0}>0
$$

and

$$
0<\liminf _{\eta \rightarrow \infty} \eta^{\ell} F(\eta) \leq \limsup _{\eta \rightarrow \infty} \eta^{\ell} F(\eta)<\infty
$$

We construct an outer supersolution in the same manner as [7, Lemma 3.1].

Lemma 3.2. Let $m+\lambda_{1}<\ell<m+\lambda_{2}+2$. Put

$$
U_{\text {out }}(r, t)=(t+\tau)^{-\ell / 2} F(\eta) \text { with } \eta=\frac{r}{(t+\tau)^{1 / 2}},
$$

where $\tau>0$ is a constant and $F$ is the positive solution obtained in Lemma 3.1. Then $U_{\text {out }}$ satisfies $\left(U_{\text {out }}\right)_{t} \geq P_{\alpha} U_{\text {out }}$ for all $t>0$ and $r>0$.

Recall that $\psi$ and $\Psi$ denote the solutions of (2.8) and (2.9), respectively.

Lemma 3.3. Let $m+\lambda_{1}<\ell<m+\lambda_{2}+2$, and set

$$
U_{\text {in }}(r, t)=(t+\tau)^{-q} \psi(r)-q(t+\tau)^{-q-1} \Psi(r),
$$

where $q=\left(\ell-m-\lambda_{1}\right) / 2$ and $\tau>0$ is a constant. Define $U_{\text {out }}$ by (3.3). Then there are positive constants $B, C_{0}, \tau_{0}$ and $R_{0}$ with $R_{0}<B \tau_{0}^{1 / 2}$ such that, for $\tau \geq \tau_{0}$, the following inequalities hold:

(i) $\left(U_{\text {in }}\right)_{t} \geq P_{\alpha} U_{\text {in }}$ for all $t>0$ and $r>0$.

(ii) $U_{\text {in }}(r, t)>0$ for all $t \geq 0$ and $r \in\left[0, B(t+\tau)^{1 / 2}\right]$.

(iii) $U_{\text {in }}(r, t)>C_{0} U_{\text {out }}(r, t)$ at $r=B(t+\tau)^{1 / 2}$ for all $t \geq 0$.

(iv) $U_{\text {in }}(r, t)<C_{0} U_{\text {out }}(r, t)$ for all $t \geq 0$ and $r \in\left[0, R_{0}\right]$.

Proof. For the proof of (i)-(iii), see [3, Lemma 3.2] and [7, Lemma 3.2]. We will show (iv). Put

$$
C_{B}=\inf \left\{\eta^{m+\lambda_{1}} F(\eta): 0<\eta \leq B\right\} .
$$

From (3.1) we have $C_{B}>0$. For all $t \geq 0$ and $r \in\left[0, B(t+\tau)^{1 / 2}\right]$, we have

$$
\begin{aligned}
C_{0} U_{\text {out }}(r, t) & =C_{0}(t+\tau)^{-\left(\ell-m-\lambda_{1}\right) / 2} r^{-m-\lambda_{1}} \eta^{m+\lambda_{1}} F(\eta) \\
& \geq C_{0} C_{B}(t+\tau)^{-\left(\ell-m-\lambda_{1}\right) / 2} r^{-m-\lambda_{1}} .
\end{aligned}
$$


Choose $R_{0} \in\left(0, B \tau^{1 / 2}\right]$ such that $C_{0} C_{B} R_{0}^{-m-\lambda_{1}}>\psi(0)$. Then, for all $t \geq 0$ and $r \in\left[0, R_{0}\right]$, we have

$$
C_{0} U_{\text {out }}(r, t)>(t+\tau)^{-\left(\ell-m-\lambda_{1}\right) / 2} \psi(0) .
$$

Since $\psi(r)$ is decreasing and $\Psi(r)$ is positive for $r>0$ by Lemma 2.3, we obtain

$$
(t+\tau)^{-\left(\ell-m-\lambda_{1}\right) / 2} \psi(0) \geq(t+\tau)^{-\left(\ell-m-\lambda_{1}\right) / 2} \psi(r) \geq U_{\text {in }}(r, t)
$$

for $r \geq 0$. Thus (iv) holds.

Assume that $B, C_{0}, \tau_{0}$ and $R_{0}$ are constants given in Lemma 3.3. Let $\tau \geq \tau_{0}$, and define $U_{\text {out }}$ and $U_{\text {in }}$ by (3.3) and (3.4), respectively. Put

$$
r^{*}(t)=\sup \left\{r>0: U_{\text {in }}(\rho, t)<C_{0} U_{\text {out }}(\rho, t) \text { for } \rho \in[0, r)\right\} .
$$

By Lemma 3.3, the function $r^{*}(t)$ is well-defined and satisfies

$$
r^{*}(t) \in\left(R_{0}, B(t+\tau)^{1 / 2}\right) \text { for all } t \geq 0 .
$$

For each $t \geq 0$, define

$$
U^{+}(r, t)= \begin{cases}U_{\text {in }}(r, t) & \text { for } 0 \leq r \leq r^{*}(t), \\ C_{0} U_{\text {out }}(r, t) & \text { for } r>r^{*}(t)\end{cases}
$$

We will show the following results.

Lemma 3.4. Let $\ell^{\prime} \in[0, \ell$ ). Then the following (i) and (ii) hold.

(i) The function $U^{+}$satisfies

$$
\left\|U^{+}(|\cdot|, t)\right\|_{\ell^{\prime}}=O\left(t^{-v}\right) \text { as } t \rightarrow \infty,
$$

where $v$ is the constant defined by (1.7).

(ii) For any constants $c>0$ and $\mu \geq 1 / 2$, the function $U^{+}$satisfies

$$
\limsup _{t \rightarrow \infty} t^{\left(\ell-\ell^{\prime}\right) \mu}\left(\sup _{|x| \geq c t^{\mu}}(1+|x|)^{\ell^{\prime}}\left|U^{+}(|x|, t)\right|\right)<\infty .
$$

Proof. (i) First, we consider the case $\ell^{\prime} \in\left(0, m+\lambda_{1}\right]$. We will show that

$$
\sup _{r \geq r^{*}(t)}(1+r)^{\ell^{\prime}} U^{+}(r, t)=O\left(t^{-\left(\ell-m-\lambda_{1}\right) / 2}\right) \quad \text { as } t \rightarrow \infty
$$

and

$$
\sup _{0<r \leq r^{*}(t)}(1+r)^{\ell^{\prime}} U^{+}(r, t)=O\left(t^{-\left(\ell-m-\lambda_{1}\right) / 2}\right) \quad \text { as } t \rightarrow \infty .
$$

For each fixed $t>0$, from (3.5) we have,

$$
\sup _{r \geq r^{*}(t)}(1+r)^{\ell^{\prime}} U^{+}(r, t) \leq \sup _{r \geq R_{0}}(1+r)^{m+\lambda_{1}} C_{0} U_{\text {out }}(r, t) \leq \sup _{r \geq R_{0}} C r^{m+\lambda_{1}} U_{\text {out }}(r, t)
$$


with some constant $C>0$. Observe that

$$
r^{m+\lambda_{1}} U_{\text {out }}(r, t)=(t+\tau)^{-\left(\ell-m-\lambda_{1}\right) / 2} \eta^{m+\lambda_{1}} F(\eta) \quad \text { with } \eta=\frac{r}{(t+\tau)^{1 / 2}} .
$$

It follows from (3.1) and (3.2) that $\sup _{\eta>0} \eta^{m+\lambda_{1}} F(\eta)<\infty$. Then we obtain (3.9). For each $t>0$, we have,

$$
\begin{aligned}
\sup _{0 \leq r \leq r^{*}(t)}(1+r)^{\ell^{\prime}} U^{+}(r, t) & \leq \sup _{r \geq 0}(1+r)^{\ell^{\prime}} U_{\text {in }}(r, t) \\
& \leq \sup _{r \geq 0}(t+\tau)^{-\left(\ell-m-\lambda_{1}\right) / 2}(1+r)^{\ell^{\prime}} \psi(r) .
\end{aligned}
$$

Since $(1+r)^{\ell^{\prime}} \psi(r)$ is bounded for $r \geq 0$ by (2.10), we obtain (3.10). Combining (3.9) and (3.10), we obtain (3.7) with $v=\left(\ell-m-\lambda_{1}\right) / 2$.

Next, we consider the case $\ell^{\prime} \in\left(m+\lambda_{1}, \ell\right)$. For each fixed $t>0$, we have

$$
\sup _{r \geq r^{*}(t)}(1+r)^{\ell^{\prime}} U^{+}(r, t) \leq \sup _{r \geq R_{0}}(1+r)^{\ell^{\prime}} C_{0} U_{\text {out }}(r, t) \leq \sup _{r \geq R_{0}} C r^{\ell^{\prime}} U_{\text {out }}(r, t)
$$

with some constant $C>0$. Observe that

$$
r^{\ell^{\prime}} U_{\text {out }}(r, t)=(t+\tau)^{-\left(\ell-\ell^{\prime}\right) / 2} \eta^{\ell^{\prime}} F(\eta) \quad \text { with } \eta=\frac{r}{(t+\tau)^{1 / 2}} .
$$

It follows from (3.2) that $\sup _{\eta>0} \eta^{\ell^{\prime}} F(\eta)<\infty$. Then

$$
\sup _{r \geq r^{*}(t)}(1+r)^{\ell^{\prime}} U^{+}(r, t)=O\left(t^{-\left(\ell-\ell^{\prime}\right) / 2}\right) \quad \text { as } t \rightarrow \infty .
$$

On the other hand, for each $t>0$, we have

$$
\begin{aligned}
\sup _{0 \leq r \leq r^{*}(t)}(1+r)^{\ell^{\prime}} U^{+}(r, t) & \leq \sup _{0 \leq r \leq B(t+\tau)^{1 / 2}}(1+r)^{\ell^{\prime}} U_{\text {in }}(r, t) \\
& \leq \sup _{0 \leq r \leq B(t+\tau)^{1 / 2}}(t+\tau)^{-\left(\ell-m-\lambda_{1}\right) / 2}(1+r)^{\ell^{\prime}} \psi(r) .
\end{aligned}
$$

By virtue of (2.10), there exist a positive constant $C$ satisfying

$$
(1+r)^{\ell^{\prime}} \psi(r) \leq C\left(1+r^{\ell^{\prime}-m-\lambda_{1}}\right) \text { for } r \geq 0 .
$$

Then it follows that

$$
\sup _{0 \leq r \leq B(t+\tau)^{1 / 2}}(1+r)^{\ell^{\prime}} \psi(r) \leq C \sup _{0 \leq r \leq B(t+\tau)^{1 / 2}}\left(1+r^{\ell^{\prime}-m-\lambda_{1}}\right)=O\left((t+\tau)^{\left(\ell^{\prime}-m-\lambda_{1}\right) / 2}\right)
$$

as $t \rightarrow \infty$. Thus, from (3.12), we obtain

$$
\sup _{0<r \leq r^{*}(t)}(1+r)^{\ell^{\prime}} U^{+}(r, t)=O\left(t^{-\left(\ell-\ell^{\prime}\right) / 2}\right) \quad \text { as } t \rightarrow \infty .
$$

Combining (3.11) and (3.13), we obtain (3.7) with $v=\left(\ell-\ell^{\prime}\right) / 2$. 
(ii) First we show that

$$
\sup _{r \geq c t^{\mu}}(1+r)^{\ell^{\prime}} U_{\text {out }}(r, t)=O\left(t^{-\left(\ell-\ell^{\prime}\right) \mu}\right) \quad \text { as } t \rightarrow \infty
$$

for any constants $c>0$ and $\mu \geq 1 / 2$. For $r \geq 1$, we have

$$
(1+r)^{\ell^{\prime}} U_{\text {out }}(r, t) \leq C r^{\ell^{\prime}}(t+\tau)^{-\ell / 2} F(\eta)=C r^{-\left(\ell-\ell^{\prime}\right)} \eta^{\ell} F(\eta)
$$

with some constant $C>1$. Note here that $r^{-\left(\ell-\ell^{\prime}\right)} \leq c^{-\left(\ell-\ell^{\prime}\right)} t^{-\left(\ell-\ell^{\prime}\right) \mu}$ for $r \geq c t^{\mu}$. Thus, from (3.2), we obtain (3.14).

In the case $\mu>1 / 2$, from (3.5), there exists $t_{0} \geq 0$ such that $c t^{\mu}>r^{*}(t)$ for $t \geq t_{0}$. Thus, from (3.14), we obtain

$$
\sup _{r \geq c t^{\mu}}(1+r)^{\ell^{\prime}} U^{+}(r, t)=\sup _{r \geq c t^{\mu}}(1+r)^{\ell^{\prime}} C_{0} U_{\text {out }}(r, t)=O\left(t^{-\left(\ell-\ell^{\prime}\right) \mu}\right) \quad \text { as } t \rightarrow \infty .
$$

In the case $\mu=1 / 2$, we observe that

$$
\begin{aligned}
& \sup _{r \geq c t^{\mu}}(1+r)^{\ell^{\prime}} U^{+}(r, t) \\
& \quad=\max \left\{\sup _{c t^{\mu} \leq r \leq r^{*}(t)}(1+r)^{\ell^{\prime}} U_{\text {in }}(r, t), \sup _{r \geq r^{*}(t)}(1+r)^{\ell^{\prime}} C_{0} U_{\text {out }}(r, t)\right\},
\end{aligned}
$$

if $c t^{\mu}<r^{*}(t)$, and that

$$
\sup _{r \geq c t^{\mu}}(1+r)^{\ell^{\prime}} U^{+}(r, t) \leq \sup _{r \geq r^{*}(t)}(1+r)^{\ell^{\prime}} C_{0} U_{\text {out }}(r, t)
$$

if $c t^{\mu} \geq r^{*}(t)$. Thus, in the case $\mu=1 / 2$, we may assume that $c t^{\mu}<r^{*}(t)$ for $t \geq t_{0}$ with some $t_{0} \geq 0$. From $c t^{\mu}<r^{*}(t)$, we have

$$
\sup _{r \geq r^{*}(t)}(1+r)^{\ell^{\prime}} U_{\text {out }}(r, t) \leq \sup _{r \geq c t^{\mu}}(1+r)^{\ell^{\prime}} U_{\text {out }}(r, t) \quad \text { for } t \geq t_{0} .
$$

Thus, from (3.14), we obtain

$$
\sup _{r \geq r^{*}(t)}(1+r)^{\ell^{\prime}} U_{\text {out }}(r, t)=O\left(t^{-\left(\ell-\ell^{\prime}\right) \mu}\right) \quad \text { as } t \rightarrow \infty .
$$

From (3.5) it follows that

$$
\sup _{c t^{\mu} \leq r \leq r^{*}(t)}(1+r)^{\ell^{\prime}} U_{\text {in }}(r, t) \leq \sup _{c t^{\mu} \leq r \leq B(t+\tau)^{1 / 2}}(1+r)^{\ell^{\prime}}(t+\tau)^{-\left(\ell-m-\lambda_{1}\right) / 2} \psi(r)
$$

for $t \geq t_{0}$. Since $\psi(r)$ is decreasing and satisfies (2.10) by Lemma 2.3, we have $\psi(r) \leq \psi\left(c t^{\mu}\right)$ for $r \geq c t^{\mu}$ and

$$
\psi\left(c t^{\mu}\right)=O\left(t^{-\left(m+\lambda_{1}\right) \mu}\right) \quad \text { as } t \rightarrow \infty .
$$

Recall that $\mu=1 / 2$. Then it follows that

$$
\sup _{c t^{\mu} \leq r \leq B(t+\tau)^{1 / 2}}(1+r)^{\ell^{\prime}}(t+\tau)^{-\left(\ell-m-\lambda_{1}\right) / 2} \psi(r)=O\left(t^{-\left(\ell-\ell^{\prime}\right) \mu}\right) \quad \text { as } t \rightarrow \infty .
$$


Thus, from (3.17), we obtain

$$
\sup _{c t^{\mu} \leq r \leq r^{*}(t)}(1+r)^{\ell^{\prime}} U_{\text {in }}(r, t)=O\left(t^{-\left(\ell-\ell^{\prime}\right) \mu}\right) \quad \text { as } t \rightarrow \infty .
$$

From (3.15) with (3.16) and (3.18), we obtain

$$
\sup _{r \geq c t^{\mu}}(1+r)^{\ell^{\prime}} U^{+}(r, t)=O\left(t^{-\left(\ell-\ell^{\prime}\right) \mu}\right) \quad \text { as } t \rightarrow \infty .
$$

Thus we obtain (3.8).

Proof of Theorem 1.1. Put $U_{0}(r)=\max _{r=|x|}\left|u_{0}(x)-\tilde{u}_{0}(x)\right|$ for $r \geq 0$, and let $U$ be a solution of (2.7). Then, by Lemma 2.4 (i), we obtain (2.11).

Define $U^{+}$by (3.6). By the definition of $r^{*}(t)$, we see that $U_{\text {in }}=C_{0} U_{\text {out }}$ and $\partial U_{\text {in }} / \partial r \geq \partial\left(C_{0} U_{\text {out }}\right) / \partial r$ at $(r, t)=\left(r^{*}(t), t\right)$ for all $t \geq 0$. Thus, if there exists a constant $C>0$ such that $C U^{+}(|x|, 0) \geq U_{0}(x)$ for $x \in \mathbf{R}^{N}$, then $C U^{+}$is a continuous weak supersolution to (2.7) by Lemma 2.2 (i).

Observe that $r^{\ell} U_{\text {out }}(r, 0)=\eta^{\ell} F(\eta)$ with $\eta=r / \tau^{1 / 2}$. Then, by (3.2), we see that

$$
\liminf _{r \rightarrow \infty} r^{\ell} U^{+}(r, 0)=C_{0} \liminf _{\eta \rightarrow \infty} \eta^{\ell} F(\eta)>0 .
$$

From (1.5) the function $U_{0}$ satisfies $\limsup _{r \rightarrow \infty} r^{\ell} U_{0}(r)<\infty$. Since $U^{+}(r, 0)>0$ for $r \geq 0$, there exists a constant $C_{1}>0$ such that $U_{0}(r) \leq C_{1} U^{+}(r, 0)$ for $r \geq 0$. Thus $C_{1} U^{+}(r, t)$ is a continuous weak supersolution to (2.7). Then, by applying Lemma 2.1 with $f(r, u)=p \phi_{\alpha}^{p-1}(r) u$, we obtain $0 \leq U(|x|, t) \leq C_{1} U^{+}(|x|, t)$ for $x \in \mathbf{R}$ and $t>0$. Thus, from (2.11), we obtain

$$
|u(x, t)-\tilde{u}(x, t)| \leq U(|x|, t) \leq C_{1} U^{+}(|x|, t) \text { for all } x \in \mathbf{R}^{N}, t \geq 0 .
$$

By Lemma 3.4 (i) and (ii), we obtain (1.6) and (1.8), respectively.

\section{Proof of Theorem 1.2}

In the proof of Theorem 1.2, we construct a subsolution of (2.7) by connecting inner and outer solutions. We give an inner solution $U_{\text {in }}$ in the same way as [3, Lemma 4.1].

LEMMA 4.1. Let $\ell>m+\lambda_{1}$, and put

$$
U_{\text {in }}(r, t)=(t+\tau)^{-\left(\ell-m-\lambda_{1}\right) / 2} \psi(r) .
$$

Then $U_{\text {in }}$ satisfies $\left(U_{\text {in }}\right)_{t} \leq P_{\alpha} U_{\text {in }}$ for all $t>0$ and $r>0$.

We construct an outer solution by following the idea presented in [3, Lemma 4.2]. 
Lemma 4.2. Let $0<\ell<N-2$, and define $U_{\text {in }}$ by (4.1). Assume that $k$ is a constant satisfying

$$
0<k<\min \left\{1, \frac{N-2-\ell}{2}\right\}
$$

Put

$$
U_{\text {out }}(r, t)=\max \left\{0, r^{-\ell}-b^{2 k}(t+\tau)^{k} r^{-\ell-2 k}\right\} \quad \text { with } b=\left(\frac{\ell(N-2-\ell)}{k}\right)^{1 / 2}
$$

Then the following (i) and (ii) hold.

(i) In the range $r>b(t+\tau)^{1 / 2}, U_{\text {out }}(r, t)$ is positive and satisfies $\left(U_{\text {out }}\right)_{t} \leq$ $P_{\alpha} U_{\text {out }}$.

(ii) Let $B>b$. Then there exist positive constants $C_{0}$ and $\tau_{0}$ such that, for $\tau \geq \tau_{0}, \quad U_{\text {in }}(r, t)<C_{0} U_{\text {out }}(r, t)$ at $r=B(t+\tau)^{1 / 2}$ for all $t \geq 0$.

Remark 4.1. We see that $m+\lambda_{2}+2<N-2$ if $p \geq p_{c}$. In fact, we have

$$
m+\lambda_{2}+2=\frac{N+2+\sqrt{(N-2-m)^{2}-8(N-2-m)}}{2} .
$$

Recall that $N \geq 11, m+\lambda_{1} \leq(N-2) / 2$ and $\lambda_{1}>0$. Then we have $m<$ $(N-2) / 2$ and $N-2-m>(N-2) / 2>4$. Since the function $x^{2}-8 x$ is increasing for $x>4$, it follows that

$$
(N-2-m)^{2}-8(N-2-m)<(N-2)^{2}-8(N-2)<(N-6)^{2} .
$$

Thus we obtain $m+\lambda_{2}+2<N-2$.

Proof. (i) In the range $r>b(t+\tau)^{1 / 2}$, we compute

$$
\begin{aligned}
\left(U_{\text {out }}\right)_{t}-P_{\alpha} U_{\text {out }} \leq & \left(U_{\text {out }}\right)_{t}-\left(U_{\text {out }}\right)_{r r}-\frac{N-1}{r}\left(U_{\text {out }}\right)_{r} \\
= & -k b^{2 k}(t+\tau)^{k-1} r^{-\ell-2 k}+\ell(N-2-\ell) r^{-\ell-2} \\
& -b^{2 k}(\ell+2 k)(N-2-\ell-2 k)(t+\tau)^{k} r^{-\ell-2-2 k} .
\end{aligned}
$$

From (4.2) and $r /(t+\tau)^{1 / 2}>b$, it follows that

$$
\begin{aligned}
\left(U_{\text {out }}\right)_{t}-P_{\alpha} U_{\text {out }} & \leq-k b^{2 k}(t+\tau)^{k-1} r^{-\ell-2 k}+\ell(N-2-\ell) r^{-\ell-2} \\
& =\left(-k b^{2 k}\left(r /(t+\tau)^{1 / 2}\right)^{2-2 k}+\ell(N-2-\ell)\right) r^{-\ell-2} \\
& \leq\left(-k b^{2}+\ell(N-2-\ell)\right) r^{-\ell-2}=0 .
\end{aligned}
$$

Thus we obtain $\left(U_{\text {out }}\right)_{t} \leq P_{\alpha} U_{\text {out }}$.

(ii) At $r=B(t+\tau)^{1 / 2}$, we have

$$
U_{\text {out }}(r, t)=B^{-\ell-2 k}\left(B^{2 k}-b^{2 k}\right)(t+\tau)^{-\ell / 2}>0
$$


and

$$
U_{\text {in }}(r, t)=(t+\tau)^{-\left(\ell-m-\lambda_{1}\right) / 2} \psi\left(B(t+\tau)^{1 / 2}\right)
$$

for all $t \geq 0$. From (2.10) there exist $r_{0}>0$ and $c>0$ such that

$$
\psi(r) \leq c r^{-m-\lambda_{1}} \quad \text { for } r \geq r_{0} .
$$

Then there exists $\tau_{0}>0$ such that, for $\tau \geq \tau_{0}$, we have $B \tau^{1 / 2}>r_{0}$ and

$$
U_{\text {in }}(r, t) \leq c B^{-m-\lambda_{1}}(t+\tau)^{-\ell / 2} \text { at } r=B(t+\tau)^{1 / 2} \text { for all } t \geq 0 .
$$

From (4.4) and (4.5), there exists $C_{0}>0$ such that $U_{\text {in }}(r, t)<C_{0} U_{\text {out }}(r, t)$ at $r=B(t+\tau)^{1 / 2}$ for all $t \geq 0$.

Assume that $B, C_{0}$ and $\tau_{0}$ are constants given in Lemma 4.2. Let $\tau \geq \tau_{0}$, and define $U_{\text {in }}$ and $U_{\text {out }}$ by (4.1) and (4.3), respectively. Put

$$
r^{*}(t)=\sup \left\{r>0: U_{\text {in }}(\rho, t)<C_{0} U_{\text {out }}(\rho, t) \text { for } \rho \in[0, r)\right\} .
$$

By Lemma 4.2, the function $r^{*}(t)$ is well-defined and satisfies

$$
r^{*}(t) \in\left(b(t+\tau)^{1 / 2}, B(t+\tau)^{1 / 2}\right) \text { for all } t \geq 0 .
$$

For each $t \geq 0$, define

$$
U^{-}(r, t)= \begin{cases}U_{\text {in }}(r, t) & \text { for } 0 \leq r \leq r^{*}(t), \\ C_{0} U_{\text {out }}(r, t) & \text { for } r>r^{*}(t)\end{cases}
$$

We will show the following results.

Lemma 4.3. Let $\ell^{\prime} \in[0, \ell$ ). Then the following (i)-(iii) hold.

(i) There exists some constant $c>0$ such that

$$
\left\|U^{-}(|\cdot|, t)\right\|_{\ell^{\prime}} \geq c t^{-v} \text { for all } t>0,
$$

where $v$ is the constant defined by (1.7).

(ii) For any constants $c>0$ and $\mu \geq 1 / 2$, the function $U^{-}$satisfies

$$
\liminf _{t \rightarrow \infty} t^{\left(\ell-\ell^{\prime}\right) \mu}\left(\sup _{r \geq c t^{\mu}}(1+r)^{\ell^{\prime}} U^{-}(r, t)\right)>0 .
$$

(iii) For any constant $c>0$, the function $U^{-}$satisfies

$$
\liminf _{t \rightarrow \infty} t^{\left(\ell-m-\lambda_{1}\right) / 2}\left(\inf _{r \leq c t^{1 / 2}}(1+r)^{m+\lambda_{1}} U^{-}(r, t)\right)>0 .
$$

Proof. (i) We see that

$\sup _{0 \leq r \leq r^{*}(t)}(1+r)^{\ell^{\prime}} U^{-}(r, t)=\sup _{0 \leq r \leq r^{*}(t)}(1+r)^{\ell^{\prime}} U_{\text {in }}(r, t) \geq(t+\tau)^{-\left(\ell-m-\lambda_{1}\right) / 2} \psi(0)$. 
From (4.6) we have, for each $t>0$,

$$
\sup _{r \geq r^{*}(t)}(1+r)^{\ell^{\prime}} U^{-}(r, t) \geq C_{0} \sup _{r \geq B(t+\tau)^{1 / 2}} r^{\ell^{\prime}} U_{\text {out }}(r, t) .
$$

Recall that (4.4) holds at $r=B(t+\tau)^{1 / 2}$. Then it follows that

$$
\sup _{r \geq r^{*}(t)}(1+r)^{\ell^{\prime}} U^{-}(r, t) \geq C_{0} B^{\ell^{\prime}-\ell-2 k}\left(B^{2 k}-b^{2 k}\right)(t+\tau)^{-\left(\ell-\ell^{\prime}\right) / 2} .
$$

Thus, if $\ell^{\prime} \in\left[0, m+\lambda_{1}\right]$, we have

$$
\sup _{r>0}(1+r)^{\ell^{\prime}} U^{-}(r, t) \geq \sup _{0<r \leq r^{*}(t)}(1+r)^{\ell^{\prime}} U^{-}(r, t) \geq(t+\tau)^{-\left(\ell-m-\lambda_{1}\right) / 2} \psi(0),
$$

and, if $\ell^{\prime} \in\left(m+\lambda_{1}, \ell\right)$, we have

$$
\sup _{r>0}(1+r)^{\ell^{\prime}} U^{-}(r, t) \geq \sup _{r \geq r^{*}(t)}(1+r)^{\ell^{\prime}} U^{-}(r, t) \geq c(t+\tau)^{-\left(\ell-\ell^{\prime}\right) / 2}
$$

with $c=C_{0} B^{\ell^{\prime}-\ell-2 k}\left(B^{2 k}-b^{2 k}\right)$. We therefore obtain (4.8) with the constant $v$ defined by $(1.7)$.

(ii) Let $\mu \geq 1 / 2$. From (4.6), we see that

$$
\sup _{r \geq c t^{\mu}}(1+r)^{\ell^{\prime}} U^{-}(r, t) \geq \sup _{r \geq B(t+\tau)^{1 / 2}}(1+r)^{\ell^{\prime}} C_{0} U_{\text {out }}(r, t),
$$

if $c t^{\mu}<B(t+\tau)^{1 / 2}$, and that

$$
\sup _{r \geq c t^{\mu}}(1+r)^{\ell^{\prime}} U^{-}(r, t)=\sup _{r \geq c t^{\mu}}(1+r)^{\ell^{\prime}} C_{0} U_{\text {out }}(r, t),
$$

if $c t^{\mu} \geq B(t+\tau)^{1 / 2}$. Thus, we may assume that there exists $t_{0} \geq 0$ such that $c t^{\mu} \geq B(t+\tau)^{1 / 2}$ for $t \geq t_{0}$. Observe that $U_{\text {out }}$ can be written by

$$
U_{\text {out }}(r, t)=r^{-\ell}\left(1-b^{2 k}\left(\frac{t+\tau}{r^{2}}\right)^{k}\right)
$$

for $r>b(t+\tau)^{1 / 2}$. Then, for $r \geq B(t+\tau)^{1 / 2}$, we have $U_{\text {out }}(r, t) \geq c_{1} r^{-\ell}$ with $c_{1}=(1-(b / B))^{2 k}>0$. Thus we obtain

$$
\sup _{r \geq c t^{\mu}}(1+r)^{\ell^{\prime}} U_{\text {out }}(r, t) \geq \sup _{r \geq c t^{\mu}} c_{1} r^{-\left(\ell-\ell^{\prime}\right)}=c_{1} c^{-\left(\ell-\ell^{\prime}\right)} t^{-\left(\ell-\ell^{\prime}\right) \mu} .
$$

From (4.11) we obtain (4.9).

(iii) Put

$$
b^{*}=\liminf _{t \rightarrow \infty} r^{*}(t)(t+\tau)^{-1 / 2} .
$$


Then $b^{*} \geq b$ by (4.6). We will verify that $b^{*}>b$. Assume to the contrary that $b^{*}=b$. Then there exists a sequence $\left\{t_{n}\right\}$ such that $t_{n} \rightarrow \infty$ and

$$
r^{*}\left(t_{n}\right)\left(t_{n}+\tau\right)^{-1 / 2} \rightarrow b \quad \text { as } n \rightarrow \infty .
$$

By the definition of $r^{*}(t)$, we have $U_{\text {in }}\left(r^{*}(t), t\right)=C_{0} U_{\text {out }}\left(r^{*}(t), t\right)$ for all $t \geq 0$. We see that

$$
r^{*}\left(t_{n}\right)^{\ell} U_{\mathrm{in}}\left(r^{*}\left(t_{n}\right), t_{n}\right)=\left(\frac{r^{*}\left(t_{n}\right)}{\left(t_{n}+\tau\right)^{1 / 2}}\right)^{\ell-m-\lambda_{1}} r^{*}\left(t_{n}\right)^{m+\lambda_{1}} \psi\left(r^{*}\left(t_{n}\right)\right) .
$$

Then, it follows from (2.10) and (4.14) that

$$
r^{*}\left(t_{n}\right)^{\ell} U_{\text {in }}\left(r^{*}\left(t_{n}\right), t_{n}\right) \rightarrow b^{\ell-m-\lambda_{1}} c_{\alpha}>0 \quad \text { as } n \rightarrow \infty .
$$

On the other hand, it follows from (4.12) and (4.14) that

$$
r^{*}\left(t_{n}\right)^{\ell} U_{\text {out }}\left(r^{*}\left(t_{n}\right), t_{n}\right)=1-b^{2 k}\left(\frac{\left(t_{n}+\tau\right)^{1 / 2}}{r^{*}\left(t_{n}\right)}\right)^{2 k} \rightarrow 0 \quad \text { as } n \rightarrow \infty .
$$

This is a contradiction. Thus we obtain $b^{*}>b$.

We observe that

$$
\begin{aligned}
& \inf _{r \leq c t^{1 / 2}}(1+r)^{m+\lambda_{1}} U^{-}(r, t) \\
& \quad=\min \left\{\inf _{r \leq r^{*}(t)}(1+r)^{m+\lambda_{1}} U_{\text {in }}(r, t), \inf _{r^{*}(t) \leq r \leq c t^{1 / 2}}(1+r)^{m+\lambda_{1}} C_{0} U_{\text {out }}(r, t)\right\},
\end{aligned}
$$

if $c t^{1 / 2}>r^{*}(t)$, and that

$$
\inf _{r \leq c t^{1 / 2}}(1+r)^{m+\lambda_{1}} U^{-}(r, t) \geq \inf _{r \leq r^{*}(t)}(1+r)^{m+\lambda_{1}} U_{\text {in }}(r, t),
$$

if $c t^{1 / 2} \leq r^{*}(t)$. Thus we may assume that $c t^{1 / 2}>r^{*}(t)$ for $t \geq t_{0}$ with some $t_{0} \geq 0$. We will show that

$$
\inf _{r \leq r^{*}(t)}(1+r)^{m+\lambda_{1}} U_{\text {in }}(r, t) \geq c_{0}(t+\tau)^{-\left(\ell-m-\lambda_{1}\right) / 2} \text { for } t \geq t_{0}
$$

and

$$
\inf _{r^{*}(t) \leq r \leq c t^{1 / 2}}(1+r)^{m+\lambda_{1}} U_{\text {out }}(r, t) \geq c_{1} t^{-\left(\ell-m-\lambda_{1}\right) / 2} \quad \text { for } t \geq t_{1}
$$

with some constants $c_{0}, c_{1}>0$ and $t_{1} \geq t_{0}$. Combining (4.15) and (4.16), we obtain (4.10). For each $t>0$, we have

$$
\inf _{r \leq r^{*}(t)}(1+r)^{m+\lambda_{1}} U_{\text {in }}(r, t) \geq \inf _{r \geq 0}(t+\tau)^{-\left(\ell-m-\lambda_{1}\right) / 2}(1+r)^{m+\lambda_{1}} \psi(r) .
$$

From (2.10) we obtain (4.15) with some constant $c_{0}>0$. From (4.12) we see that

$$
(1+r)^{m+\lambda_{1}} U_{\text {out }}(r, t) \geq r^{m+\lambda_{1}} U_{\text {out }}(r, t)=r^{-\left(\ell-m-\lambda_{1}\right)}\left(1-b^{2 k}\left(\frac{(t+\tau)^{1 / 2}}{r}\right)^{2 k}\right) .
$$


Thus, for each $t>0$, we have

$$
\begin{aligned}
& \inf _{r^{*}(t) \leq r \leq c t^{1 / 2}}(1+r)^{m+\lambda_{1}} U_{\text {out }}(r, t) \\
& \quad \geq \inf _{r^{*}(t) \leq r \leq c t^{1 / 2}} r^{-\left(\ell-m-\lambda_{1}\right)}\left(1-b^{2 k}\left(\frac{(t+\tau)^{1 / 2}}{r^{*}(t)}\right)^{2 k}\right) .
\end{aligned}
$$

From (4.13) with $b^{*}>b$, it follows that

$$
\liminf _{t \rightarrow \infty}\left(1-b^{2 k}\left(\frac{(t+\tau)^{1 / 2}}{r^{*}(t)}\right)^{2 k}\right)=\left(1-\left(b / b^{*}\right)^{2 k}\right)>0 .
$$

Thus there exist constants $c_{2}>0$ and $t_{1} \geq t_{0}$ such that

$$
\left(1-b^{2 k}\left(\frac{(t+\tau)^{1 / 2}}{r^{*}(t)}\right)^{2 k}\right) \geq c_{2} \text { for } t \geq t_{1} .
$$

Then, from (4.17) we obtain, for $t \geq t_{1}$,

$$
\inf _{r^{*}(t) \leq r \leq c t^{1 / 2}}(1+r)^{m+\lambda_{1}} U_{\text {out }}(r, t) \geq \inf _{r^{*}(t) \leq r \leq c t^{1 / 2}} c_{2} r^{-\left(\ell-m-\lambda_{1}\right)} \geq c_{1} t^{-\left(\ell-m-\lambda_{1}\right) / 2}
$$

with $c_{1}=c_{2} c^{-\left(\ell-m-\lambda_{1}\right)}$. Thus (4.16) holds.

Proof of Theorem 1.2. Put $U_{0}(r)=\min _{r=|x|}\left(u_{0}(x)-\tilde{u}_{0}(x)\right)>0$ for $r \geq 0$, and let $U$ be a solution of (2.7). Then, by Lemma 2.4 (ii), we obtain (2.12).

Define $U^{-}$by (4.7). By Lemma 2.2 (ii) we see that, if there exists a constant $C>0$ such that $C U^{-}(|x|, 0) \leq U_{0}(x)$ for $x \in \mathbf{R}^{N}$, then $C U^{-}$is a continuous weak subsolution to (2.7). From (1.10), the function $U_{0}$ satisfies $\liminf _{r \rightarrow \infty} r^{\ell} U_{0}(r)>0$. Since $U_{0}(r)>0$ for $r \geq 0$ and

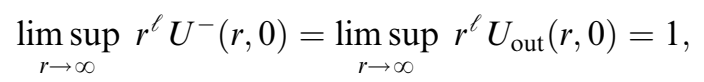

there exists a constant $C_{2}>0$ such that $C_{2} U^{-}(r, 0) \leq U_{0}(r)$ for $r \geq 0$. Then $C_{2} U^{-}(r, t)$ is a continuous weak subsolution to (2.7). Then, by Lemma 2.1, we have $U(r, t) \geq C_{2} U^{-}(r, t)$ for $r \geq 0$ and $t>0$. Thus, from (2.12), we obtain

$$
u(x, t)-\tilde{u}(x, t) \geq U(|x|, t) \geq C_{2} U^{-}(|x|, t) \text { for all } x \in \mathbf{R}^{N}, t \geq 0 .
$$

By Lemma 4.3, we obtain (1.11), (1.12) and (1.13).

\section{Proof of Theorem 1.3}

Define $U^{+}(r, t)$ as in the proof of Theorem 1.1, that is, $U^{+}(r, t)$ is defined by (3.6), where $U_{\text {out }}$ and $U_{\text {in }}$ are given by (3.3) and (3.4), respectively. Note here 
that $r^{*}(t)$ satisfies (3.5). By the similar arguments as in the proof of Lemma 3.4, we see that (3.11) and (3.13) hold even if $\ell^{\prime}=\ell$, that is,

$\sup _{r \geq r^{*}(t)}(1+r)^{\ell} U^{+}(r, t)=O(1) \quad$ and $\sup _{0<r \leq r^{*}(t)}(1+r)^{\ell} U^{+}(r, t)=O(1) \quad$ as $t \rightarrow \infty$.

Then it follows that

$$
\sup _{t \geq 0}\left(\sup _{r \geq 0}(1+r)^{\ell} U^{+}(r, t)\right)<\infty .
$$

Put $c_{1}$ and $c_{2}$, respectively, by

$$
c_{1}=\sup _{t \geq 0}\left(\sup _{r \geq 0}(1+r)^{\ell} U^{+}(r, t)\right) \text { and } c_{2}=\inf _{r \geq 0}(1+r)^{\ell} U^{+}(r, 0) .
$$

Then, by (3.19), we have $c_{2}>0$.

Lemma 5.1. For any $\varepsilon>0$, put $\delta=c_{2} \varepsilon / c>0$ with $c>c_{1}$. Let $U$ be a solution of (2.7). If $U_{0}$ satisfies $\left\|U_{0}(|\cdot|)\right\|_{\ell}<\delta$, then $U$ satisfies $\|U(|\cdot|, t)\|_{\ell}<\varepsilon$ for $t \geq 0$.

Proof. If $\left\|U_{0}(|\cdot|)\right\|_{\ell}<\delta$, then it follow that

$$
(1+r)^{\ell} U_{0}(r)<\delta \leq \frac{\varepsilon}{c}(1+r)^{\ell} U^{+}(r, 0) \text { for } r \geq 0 .
$$

This implies that $U_{0}(r) \leq(\varepsilon / c) U^{+}(r, 0)$ for $r \geq 0$. Then $(\varepsilon / c) U^{+}(r, t)$ is a continuous weak supersolution of (2.7). By Lemma 2.1 we obtain $U(r, t) \leq$ $(\varepsilon / c) U^{+}(r, t)$ for all $r \geq 0$ and $t \geq 0$. Hence,

$$
(1+r)^{\ell} U(r, t) \leq \frac{\varepsilon}{c}(1+r)^{\ell} U^{+}(r, t)<\varepsilon \text { for all } r \geq 0, t \geq 0 .
$$

Thus we obtain $\|U(|\cdot|, t)\|_{\ell}<\varepsilon$ for $t \geq 0$.

Proof of Theorem 1.3. (i) Put $U_{0}(r)=\max _{r=|x|}\left(u_{0}(x)-\tilde{u}_{0}(x)\right)$ for $r \geq 0$, and let $U$ be a solution of (2.7). Then, by Lemma 2.4 (i), we obtain (2.11).

For any $\varepsilon>0$, put $\delta=c_{2} \varepsilon / c_{1}>0$. Assume that $\left\|u_{0}(\cdot)-\tilde{u}_{0}(\cdot)\right\|_{\ell}<\delta$. This implies that $\left\|U_{0}(|\cdot|)\right\|_{\ell}<\delta$. By Lemma 5.1 we obtain $\|U(|\cdot|, t)\|_{\ell}<\varepsilon$ for $t \geq 0$. From (2.11) we conclude that $\|u(\cdot, t)-\tilde{u}(\cdot, t)\|_{\ell}<\varepsilon$ for $t \geq 0$.

(ii) Define $U^{-}(r, t)$ as in the proof of Theorem 1.2, that is, $U^{-}(r, t)$ is defined by (4.7), where $U_{\text {in }}$ and $U_{\text {out }}$ are given by (4.1) and (4.3), respectively. For each fixed $t>0$, observe that $\lim _{r \rightarrow \infty} r^{\ell} U_{\text {out }}(r, t)=1$. Then it follows that

$$
\sup _{r>0}(1+r)^{\ell} U^{-}(r, t) \geq C_{0} \text { for all } t \geq 0 .
$$

Thus we obtain $\left\|U^{-}(|\cdot|, t)\right\|_{\ell} \geq C_{0}>0$ for each $t \geq 0$. 
Put $U_{0}(r)=\min _{r=|x|}\left(u_{0}(x)-\tilde{u}_{0}(x)\right)>0$ for $r \geq 0$, and let $U$ be a solution of (2.7). Then, by Lemma 2.4 (ii), we obtain (2.12). By the similar argument as in the proof of Theorem 1.2, there exists a constant $C_{2}>0$ such that $C_{2} U^{-}(r, 0) \leq$ $U_{0}(r)$ for $r \geq 0$. Then $C_{2} U^{-}(r, t)$ is a continuous weak subsolution to (2.7). Then, by Lemma 2.1, we have $U(r, t) \geq C_{2} U^{-}(r, t)$ for $r \geq 0$ and $t>0$. Thus, from (2.11) we obtain

$$
\|u(\cdot, t)-\tilde{u}(\cdot, t)\|_{\ell} \geq\|U(|\cdot|, t)\|_{\ell} \geq C_{2}\left\|U^{-}(|\cdot|, t)\right\|_{\ell} \geq C_{2} C_{0}>0
$$

for each fixed $t>0$. This implies that (1.15) holds.

\section{REFERENCES}

[ 1 ] W. X. ChEn AND C. LI, Classification of solutions of some nonlinear elliptic equations, Duke Math. J. 63 (1991), 615-622.

[2] M. Fila, J. R. King, M. Winkler and E. Yanagida, Optimal lower bound of the grow-up rate for a supercritical parabolic equation, J. Differential Equations 228 (2006), 339-356.

[3] M. Fila, M. Winkler AND E. Yanagida, Convergence rate for a parabolic equation with supercritical nonlinearity, J. Dynam. Differential Equations 17 (2005), 249-269.

[4] B. GidAs AND J. SpRUCK, Global and local behavior of positive solutions of nonlinear elliptic equations, Commun. Pure Appl. Math. 34 (1981), 525-598.

[5] C. GuI, W.-M. Ni AND X. WANG, On the stability and instability of positive steady states of a semilinear heat equation in $R^{n}$, Comm. Pure Appl. Math. 45 (1992), 1153-1181.

[6] C. GuI, W.-M. Ni AND X. WANG, Further study on a nonlinear heat equation, J. Differential Equations 169 (2001), 588-613.

[7] M. Hoshino AND E. Yanagida, Sharp estimates of the convergence rate for a semilinear parabolic equation with supercritical nonlinearity, Nonlinear Anal. TMA 69 (2008), 3136-3152.

[8] D. D. Joseph AND T. S. Lundgren, Quasilinear Dirichlet problems driven by positive sources, Arch. Rational Mech. Anal. 49 (1972/73), 241-269.

[9] Y. NAITO, Global attractivity and convergence rate in the weighted norm for a supercritical semilinear heat equation, to appear in Differential Integral Equations.

[10] P. PoláčIK And E. Yanagida, On bounded and unbounded global solutions of a supercritical semilinear heat equation, Math. Ann. 327 (2003), 745-771.

[11] X. WANG, On the Cauchy problem for reaction-diffusion equations, Trans. Amer. Math. Soc. 337 (1993), 549-590.

Yūki Naito

Department of Mathematics

EHIME UNIVERSITY

MATSUYAMA 790-8577

JAPAN

E-mail: ynaito@ehime-u.ac.jp 